\title{
Long-Term Paired Associative Stimulation Enhances Motor Output of the Tetraplegic Hand
}

\author{
Aleksandra Tolmacheva, Sarianna Savolainen, ${ }^{2}$ Erika Kirveskari, ${ }^{3}$ Pantelis Lioumis, ${ }^{1}$ Linda Kuusela, ${ }^{4,5}$ \\ Nina Brandstack, Aarne Ylinen, Jyrki P. Mäkelä, and Anastasia Shulga ${ }^{1,6}$
}

\begin{abstract}
A large proportion of spinal cord injuries (SCI) are incomplete. Even in clinically complete injuries, silent non-functional connections can be present. Therapeutic approaches that can strengthen transmission in weak neural connections to improve motor performance are needed. Our aim was to determine whether long-term delivery of paired associative stimulation (PAS, a combination of transcranial magnetic stimulation [TMS] with peripheral nerve stimulation [PNS]) can enhance motor output in the hands of patients with chronic traumatic tetraplegia, and to compare this technique with long-term PNS. Five patients (4 males; age 38-68, mean 48) with no contraindications to TMS received 4 weeks (16 sessions) of stimulation. PAS was given to one hand and PNS combined with sham TMS to the other hand. Patients were blinded to the treatment. Hands were selected randomly. The patients were evaluated by a physiotherapist blinded to the treatment. The follow-up period was 1 month. Patients were evaluated with Daniels and Worthingham's Muscle Testing (0-5 scale) before the first stimulation session, after the last stimulation session, and 1 month after the last stimulation session. One month after the last stimulation session, the improvement in the PAS-treated hand was $1.02 \pm 0.17$ points ( $p<0.0001, n=100$ muscles from 5 patients). The improvement was significantly higher in PAS-treated than in PNS-treated hands (176 $\pm 29 \%, p=0.046, n=5$ patients). Longterm PAS might be an effective tool for improving motor performance in incomplete chronic SCI patients. Further studies on PAS in larger patient cohorts, with longer stimulation duration and at earlier stages after the injury, are warranted.
\end{abstract}

Keywords: paired associative stimulation; peripheral electrical stimulation; plasticity; spinal cord injury; transcranial magnetic stimulation

\section{Introduction}

A LARGE PROPORTION of spinal cord injuries (SCI) are incomplete. ${ }^{1}$ SCI patients may also have preserved non-functional connections after clinically complete injuries. ${ }^{2}$ Strengthening residual pathways in incomplete SCI patients through a wide range of non-invasive methods has gained considerable attention in human SCI research. ${ }^{3,4}$ Long-term potentiation (LTP), depending on the cooperativity and associativity of neuronal activation, ${ }^{5}$ has been one of the central targets in counteracting the connectivity weakness after neuronal trauma and disease. Indeed, evidence from animal studies indicates that stimulation protocols inducing spiketime-dependent-like plasticity between upper and lower motor neurons are promising tools for strengthening the residual connectivity and promoting motor recovery. ${ }^{6-8}$
Paired associative stimulation (PAS), ${ }^{9}$ a combination of transcranial magnetic stimulation (TMS) with peripheral nerve stimulation (PNS), is known to induce LTP-like plasticity in human motor $^{10}$ and sensory ${ }^{11}$ tracts through synchronized activation of upper motor neurons and peripheral neurons in healthy individuals. ${ }^{12}$ PAS-induced plasticity is physiologically relevant, as it correlates with an increase in muscle voluntary force. ${ }^{13}$ Singlesession PAS targeting the level of corticomotoneuronal synapses of the spinal cord induces transient plastic changes in healthy subjects and in incomplete SCI patients. ${ }^{14}$

The TMS-PNS combination is clinically advantageous as it is non-invasive and the required equipment is available in hospitals and research laboratories worldwide. Both stimulation modalities are safe and are in clinical use. ${ }^{15-17}$ PNS is used for improving muscle strength, increasing range of motion, reducing edema, and

\footnotetext{
${ }^{1}$ BioMag Laboratory, ${ }^{3}$ Clinical Neurosciences, Clinical Neurophysiology, ${ }^{5}$ Department of Radiology, HUS Medical Imaging Center, University of Helsinki and Helsinki University Hospital, Helsinki, Finland.

${ }^{2}$ Validia Rehabilitation Center, Helsinki, Finland.

${ }^{4}$ Department of Physics, University of Helsinki, Helsinki, Finland.

${ }^{6}$ Clinical Neurosciences, Neurology, University of Helsinki and Helsinki University Hospital, Helsinki, Finland.

(CAleksandra Tolmacheva et al., 2017; Published by Mary Ann Liebert, Inc. This Open Access article is distributed under the terms of the Creative Commons Attribution Noncommercial License (http://creativecommons.org/licenses/by-nc/4.0/) which permits any noncommercial use, distribution, and reproduction in any medium, provided the original author(s) and the source are credited.
} 
decreasing atrophy and pain. ${ }^{18}$ Various peripheral electrical stimulation techniques, such as transcutaneous electrical nerve stimulation (TENS), neuromuscular electrical stimulation (NMES), and functional electrical stimulation (FES) are widely used and are under intense investigation. ${ }^{18}$ Nevertheless, for SCI patients, only TENS has been identified as an effective treatment for reducing pain, with moderate strength of evidence. ${ }^{19}$ Little information is available on the therapeutic potential of PNS combined with TMS when applied over a long period of time. Thus far, only two reports describe the effects of long-term PAS in neurological patients. ${ }^{20,21}$

A combination of different types of stimulation is increasingly used in animal and human research. It is essential to determine any possible clinically relevant advantages of paired versus unpaired stimulation. $^{22}$ To our knowledge, no reports have compared longterm PAS with peripheral stimulation only.

The therapeutic potential of long-term PAS for SCI patients is unexplored. We have recently applied a PAS protocol with novel settings $^{23}$ in a long-term way to two incomplete SCI patients, ${ }^{20}$ providing the first demonstration of the therapeutic potential of long-term PAS for this patient group.

Here we confirm these pilot results in a systematic study, and for the first time compare long-term PAS with long-term PNS.

\section{Methods}

\section{Patients}

The protocol (Fig. 1) was approved by the Ethics Committee of Medicine of the Helsinki University Hospital. All patients provided informed written consent. Five patients (4 males; age 38-68, mean 48; Table 1) with incomplete chronic traumatic tetraplegia participated in the study. All patients fully adhered to the protocol. The individual conventional rehabilitation (Table 1) of all patients was continued in parallel with the study. The patients were asked to keep all exercises affecting the hands as symmetric as possible during the stimulation and follow-up periods. As part of conventional rehabilitation, Patient 5 received local peripheral stimulation to the wrists; this stimulation was on hold from 1 month before the study to the end of the follow-up period. The other patients did not receive any stimulation therapies. The medications affecting the central nervous system (CNS) are listed in Table 1. To ensure that any lack of vitamins or minerals would not be a constraint for a possible therapeutic effect, all patients were instructed to take a standard multivitamin dose during the stimulation and follow-up periods.

\section{Experimental setup}

Each patient received 16 sessions of stimulation during 4 weeks: 5 times per week during the first 2 weeks and 3 times per week thereafter (Fig. 1), corresponding to the protocol that we used for our pilot patients. ${ }^{20}$ We hypothesized that the effect of PAS might be shorter at the beginning of the stimulation series than at its end, when the connections between upper and lower motor neurons have gained strength. Patient 5 went through an additional 3 sessions (5 weeks of stimulation); the longer period was considered useful because of the low baseline motor score and advanced age. We delivered PAS to one hand and sham TMS combined with real PNS to the other hand. The stimulated hand was selected randomly (Table 2). The stimulation order (PAS or PNS hand first) was changed at every session.

\section{Evaluation of the patients}

The patients, the physiotherapist evaluating motor and spasticity scores, and the clinical neurophysiologist evaluating F-responses were blinded to the treatment. Sensory scores were evaluated by an unblinded physician.

\section{Motor scores}

An experienced physiotherapist performed manual motor testing $^{24}$ (Supplementary Table 1; see online supplementary material at http://www.liebertpub.com) of each muscle with other muscles manually immobilized. The first evaluation was done before, the second immediately after, and the third 1 month after the 4-week intervention (Fig. 1). We calculated the differences between the first and second evaluation (change during 4-week stimulation), the second and third evaluation (change during the follow-up period),

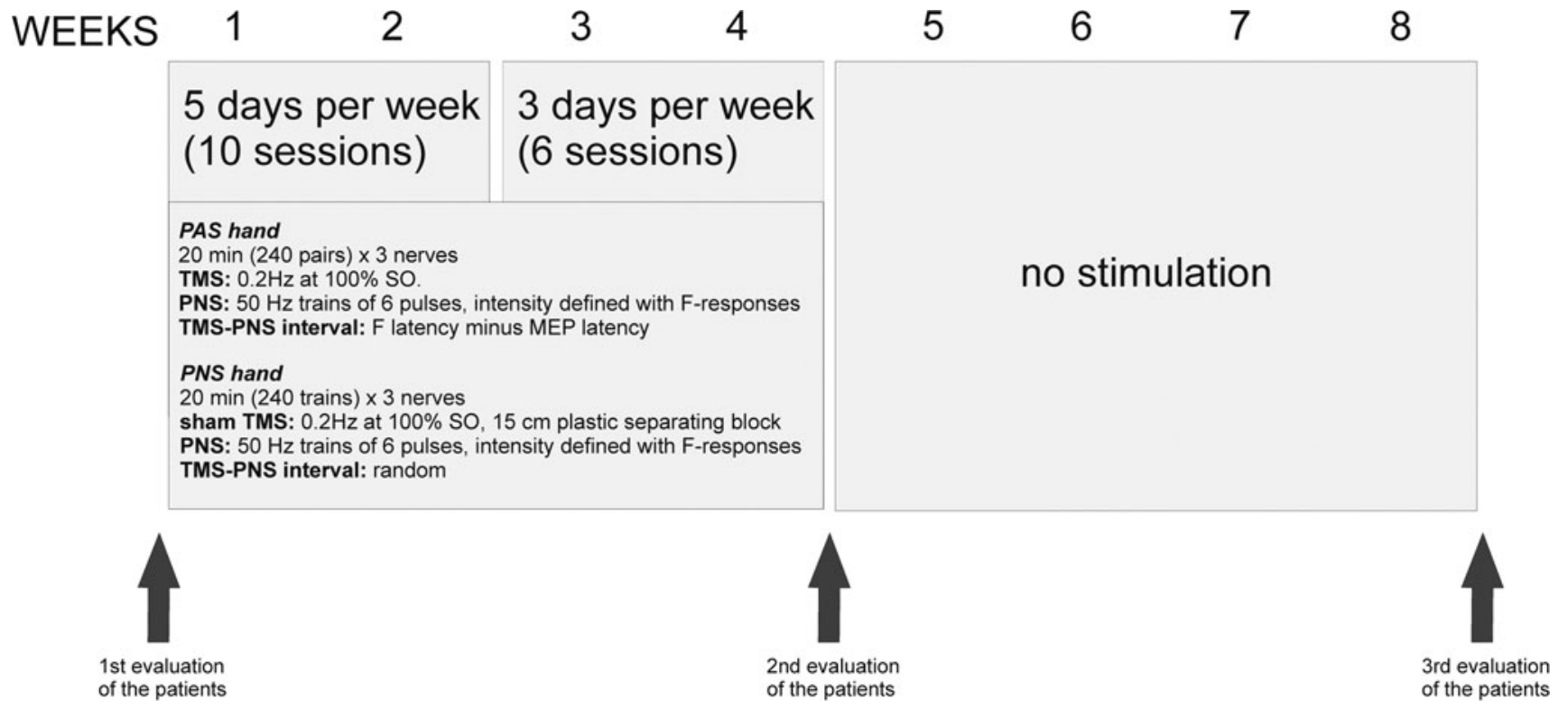

FIG. 1. Time line of the experiment and the summary of the stimulation protocol. MEP, motor-evoked potential; PAS, paired associative stimulation; PNS, peripheral nerve stimulation; SO, stimulator output; TMS, transcranial magnetic stimulation. 
Table 1. Detailed Patient Information

\begin{tabular}{|c|c|c|c|c|c|c|c|c|}
\hline Patient & Gender & age & $\begin{array}{l}\text { Neuro- } \\
\text { logical } \\
\text { level }\end{array}$ & $A I S$ & $\begin{array}{l}\text { Time since } \\
\text { injury (years } \\
\text { and months) }\end{array}$ & $\begin{array}{l}\text { Height } \\
(\mathrm{cm})\end{array}$ & $\begin{array}{l}\text { Conventional rehabilitation } \\
\text { (times per week) }\end{array}$ & Medication affecting CNS \\
\hline 1 & Male & 38 & $\mathrm{C} 7$ & $\mathrm{~B}$ & $4 y, 3 \mathrm{~m}$ & 180 & $\begin{array}{l}\text { Physiotherapy }(1 \mathrm{~h} \times 2) \text {, } \\
\text { occupational therapy }(1 \mathrm{~h} \times 2) \text {, } \\
\text { swimming pool }(1 \mathrm{~h} \times 2)\end{array}$ & $\begin{array}{l}\text { Baclofen } 25 \mathrm{mg} \times 3 \\
\text { clonazepam } 1 \mathrm{mg} \times 3 \\
\text { (on demand up to } 10 \mathrm{mg} \text { per day) }\end{array}$ \\
\hline 2 & Male & 38 & $\mathrm{C} 7$ & $\mathrm{D}$ & $5 \mathrm{y}, 10 \mathrm{~m}$ & 190 & $\begin{array}{l}\text { Physiotherapy }(1 \mathrm{~h} \times 2) \text {, } \\
\text { occupational therapy }(1 \mathrm{~h} \times 1) \text {, } \\
\text { swimming pool }(1 \mathrm{~h} \times 1)\end{array}$ & $\begin{array}{l}\text { Baclofen } 25 \mathrm{mg} \times 2 \\
\quad \text { clonazepam } 0.5 \mathrm{mg} \times 1\end{array}$ \\
\hline 3 & Male & 42 & $\mathrm{C} 4$ & $\mathrm{C}$ & $6 y, 7 \mathrm{~m}$ & 174 & Physiotherapy $(1-2 \mathrm{~h} \times 1)$ & $\begin{array}{l}\text { Pregabalin } 75 \mathrm{mg}+225 \mathrm{mg}, \\
\text { baclofen } 25 \mathrm{mg} \times 2 \\
\text { tizanidine } 6 \mathrm{mg} \times 2\end{array}$ \\
\hline 4 & Male & 53 & $\mathrm{C} 3$ & $\mathrm{C}$ & $3 \mathrm{y}, 11 \mathrm{~m}$ & 174 & $\begin{array}{l}\text { Physiotherapy }(1 \mathrm{~h} \times 2) \text {, } \\
\text { occupational therapy }(1 \mathrm{~h} \times 2) \text {, } \\
\text { swimming pool }(1 \mathrm{~h} \times 1)\end{array}$ & $\begin{array}{l}\text { Mirtazapin } 15 \mathrm{mg} \times 1 \\
\text { temazepam } 5 \mathrm{mg} \times 1 \\
\text { clonazepam } 1 \mathrm{mg} \times 2 \\
\text { baclofen } 20 \mathrm{mg} \times 3\end{array}$ \\
\hline 5 & Female & 68 & $\mathrm{C} 5$ & $\mathrm{C}$ & $1 \mathrm{y}$ & 164 & $\begin{array}{l}\text { Physiotherapy }(1 \mathrm{~h} \times 1) \text {, } \\
\text { swimming pool }(1 \mathrm{~h} \times 1), \\
\text { occupational therapy }(1 \mathrm{~h} \times 1) \\
\text { per month }\end{array}$ & $\begin{array}{l}\text { Amitriptylline } 25 \mathrm{mg} \times 1 \text {, } \\
\text { gabapentin } 300 \mathrm{mg}+900 \mathrm{mg}+ \\
900 \mathrm{mg} \text {, clonazepam } 1 \mathrm{mg} \times 1\end{array}$ \\
\hline
\end{tabular}

AIS, American Spinal Injury Association Impairment Scale; CNS, central nervous system.

and the first and third evaluation (total change) for each muscle. The differences were averaged for the PAS and PNS hand of each patient, and the PAS-to-PNS ratio was counted from the averages (Supplementary Table 1).

The physiotherapist did not have access to the results of the previous evaluations. The muscles with a value of 5 before intervention (full strength) were not included in the analysis of the therapeutic effects. In addition, the muscles of the right thumb of Patient 3 were excluded from the analysis, because the thumb ligaments were injured before SCI. The full list of muscles evaluated in each patient is provided in Table 3.

\section{Spasticity}

An experienced physiotherapist evaluated spasticity by a modified Asworth Scale from elbow (extensors, flexors), wrist (extensors, flexors), hip (flexors, extensors, adductors), knee (flexors, extensors), and ankle (plantar flexors) joints.

Motor evoked potentials (MEPs) were measured to the stimulation of each hemisphere, but the amplitudes and latencies could not be reliably evaluated due to background spasticity. In four patients, we analyzed the number of spasticity-related spikes from the electromyography (EMG) derived from MEP recordings. For each patient, 500-msec spontaneous EMG periods recorded im- mediately before the 15 TMS pulses, delivered with 3.3-sec intervals (altogether $7.5 \mathrm{sec}$ ) were analyzed: from abductor pollicis brevis (APB), abductor digiti minimi (ADM), and brachioradialis (BR). The number of motor unit potentials exceeding the baseline EMG was calculated manually from the recordings. We ensured that the baseline was equal in all recordings.

\section{Transcranial magnetic stimulation (TMS)}

We administered TMS with an eXimia magnetic stimulator (Nexstim Ltd., Helsinki, Finland) with a figure-of-eight coil. The device incorporates a magnetic resonance imaging (MRI)-based navigation system. Optimal stimulation sites for APB, ADM, and BR were defined by mapping the primary motor cortex. Spasticityrelated EMG activity and movement artifacts resulting from the activation of off-target muscles with better-preserved innervation during mapping prevented an exact resting motor threshold (RMT) determination. Each patient was mapped at a wide range of stimulator output (SO) intensities; in muscles with an RMT over $100 \%$ $\mathrm{SO}$ we used muscle pre-activation, motor imagery, or both to elicit MEPs. High TMS intensities elicited MEPs from the muscles of interest more effectively, whereas at lower intensities, there were less movement artifacts. "Hot spots" were determined as sites where MEPs were most consistently elicited at a wide range of

Table 2. Stimulation Parameters and Characteristics of PAS- and PNS-treated Hands

\begin{tabular}{lccccrr}
\hline Patient & $\begin{array}{c}\text { Right- } \text { or } \\
\text { left-handed }\end{array}$ & $\begin{array}{c}\text { PAS } \\
\text { hand }\end{array}$ & $\begin{array}{c}\text { Motor score of PAS hand } \\
\text { compared with PNS hand } \\
\text { at first evaluation }\end{array}$ & $\begin{array}{c}\text { PNS intensity } \\
\text { for PAS hand } \\
(\text { mA: med, uln, rad })\end{array}$ & $\begin{array}{c}\text { PNS intensity } \\
\text { for PNS hand } \\
(\text { mA: med, uln, rad })\end{array}$ & $\begin{array}{c}\text { ISI for PAS hand, } \\
(\text { msec: med, uln, rad })\end{array}$ \\
\hline 1 & Right & Left & Stronger by 39 points & $15,15,67$ & $7,9,23$ & $1,3,3$ \\
2 & Right & Left & Weaker by 11 points & $4,8,18$ & $16,16,21$ & $5,7,6$ \\
3 & Left & Right & Weaker by 44 points & $5,8,16$ & $2,7,19$ & $-8,-9,2$ \\
4 & Right & Right & Stronger by 6 points & $4,26,22$ & $4,16,30$ & $-6,-1,-2$ \\
5 & Right & Right & Stronger by 9 points & $16,16,12$ & $18,18,17$ & $-1,1,-1$ \\
\hline
\end{tabular}

ISI, interstimulus interval; med, median; PAS, paired associative stimulation; PNS, peripheral nerve stimulation; rad, radial; uln, ulnar. 
Table 3. List of Evaluated Muscles

\section{Upper limb}

Pronator teres and quadratus

Flexor carpi radialis

Flexor digitorum superficialis

Flexor digitorum profundus (fingers II-III)

Flexor pollicis brevis

Flexor pollicis longus

Opponens pollicis

Abductor pollicis brevis

Adductor pollicis

Dorsal interossei

Abductor digiti minimi

Palmar interossei

Flexor digitorum profundus (fingers iv-v)

Flexor carpi ulnaris

Extensor digitorum

Abductor pollicis longus

Extensor pollicis longus

Extensor pollicis brevis

Extensor carpi radialis

Extensor carpi ulnaris

Brachioradialis

Supinator and biceps brachii (supination)

Triceps brachii

Serratus anterior

Upper trapezius and levator scapulae

Anterior deltoideus and supraspinatus

Middle deltoideus and supraspinatus

Posterior deltoideus

Infraspinatus and teres minor

Biceps and brachialis

Lower trapezius

Middle trapezius and rhomboids

Rhomboids

\section{Neck and body}

Neck extensors

Neck flexors

Sternocleidomastoideus

Erector trunci

Quadratus lumborum

Rectus abdominis

External and internal oblique

\section{Lower limb}

Iliopsoas

Gluteus maximus

Gluteus medius

Adductors (hip)

External rotators (hip)

Internal rotators (hip)

Semitendinosus

Biceps femoris

Quadriceps femoris

Gastrocnemius and soleus

Tibialis anterior

Tibialis posterior

Peroneus longus and brevis

Lumbricales and flexor hallucis brevis

Flexor hallucis longus

Flexor digitorum longus and brevis

Extensor digitorum longus and brevis

Extensor hallucis longus intensities. For MEP latency determination, 15 TMS pulses were administered over the selected hot spot of each muscle. Sham TMS was performed with identical stimulation parameters by placing a 15-cm plastic separating block between the TMS coil and the patient's head.

\section{Peripheral nerve stimulation (PNS)}

PNS was given with a Dantec Keypoint ${ }^{\circledR}$ electroneuromyography device (Natus Medical Inc., Pleasanton, CA) using surface electrodes (Neuroline 720; Ambu A/S, Ballerup, Denmark). We stimulated the median and ulnar nerves at the wrist, and radial nerve at the arm. For the photographs of the stimulation sites, please see the study by Shulga and colleagues. ${ }^{20}$ In radial nerve stimulation, the electrodes were manually pressed against the tissues to decrease the distance between the electrodes and the nerve; adequate stimulation was verified by observing the activation of the muscles innervated by the stimulated nerve. Stimulation intensity was individually adjusted for each nerve of each patient, and set to the minimum value reliably evoking the F-responses measured with single 1-msec pulses (see below). This was done to reliably ensure the activation of the motor fibers $^{23}$ (Table 2). PNS was administered in $50-\mathrm{Hz}$ trains consisting of 6 biphasic 1-msec square wave pulses (that is, each train consisted of one pulse every $20 \mathrm{msec}$ during $100 \mathrm{msec}$ ). ${ }^{20}$ In all patients, the stimulation elicited visible contractions of the muscles innervated by the stimulated nerves. All patients with well-preserved sensation (Patients 1-3 and 5) perceived PNS as unpleasant, especially during the first sessions. However, all patients quickly adapted to stimulation and tolerated it in subsequent sessions. Local skin anesthesia with EMLA $^{23}$ ointment prior to stimulation was offered to patients; none of them wanted the anesthesia. No patients experienced autonomic dysreflexia symptoms during the stimulation.

\section{F-response measurements}

F-wave latencies, amplitudes, and persistence were determined from 10 F-responses recorded with the Dantec Keypoint ${ }^{\circledR}$ device (see above) using surface electrodes (Neuroline 720; Ambu A/S, Ballerup, Denmark). In stimulation we used 0.2-msec pulses at supramaximal intensity (i.e., increasing the intensity did not produce an additional increase in F-wave amplitude). The stimulating electrodes were placed as for PNS (see above). The recording electrodes were placed on APB for the median nerve, on ADM for the ulnar nerve, and on BR for the radial nerve. Thereafter, to individually select PNS intensity for each patient, we determined the minimal intensity eliciting detectable F-responses using single 1-msec pulses. During the measurements the temperature of the limb was $>30^{\circ} \mathrm{C}$.

\section{PAS/PNS-only sessions}

TMS and PNS were triggered by Presentation (Neurobehavioral Systems Inc., Albany, NY) software. PAS was given at $0.2 \mathrm{~Hz}$. The interstimulus interval (ISI) between TMS and PNS was determined individually for each patient on the basis of F-response and MEP latencies as described previously. ${ }^{20,23,25} \mathrm{We}$ aimed at ISI $=0 \mathrm{msec}$, that is, simultaneous arrival of TMS-evoked activation and the activation evoked by the first pulse of PNS train at the level of the corticospinal synapses of the cervical spinal cord. During PAS, we applied TMS at $100 \%$ SO, eliciting the highest possible amount of orthodromic volleys by a single TMS pulse. ${ }^{23}$ High-intensity TMS pulses result in a high-frequency repetitive discharge of corticospinal neurons and this ensures multiple collisions of pre- and post-synaptic pulses at the spinal cord level. ${ }^{26}$ For sham TMS, we used $100 \%$ SO to ensure a similar noise level. Each 20-min PAS or PNS-only session comprised 240 PAS pulses or 240 PNS-only trains. During both PAS and PNS sessions, the patients were instructed to concentrate on the movement produced by the muscles 
innervated by the stimulated nerve. We hypothesized that motor imagery during PAS sessions lowers the RMT of the corresponding cortical representation area and thus increases the number of corticospinal volleys elicited by each TMS pulse. Each experimental session took $2 \mathrm{~h}$ (PAS hand: $20 \mathrm{~min} \times 3$ nerves and PNS hand: $20 \min \times 3$ nerves), excluding the time for preparation.

\section{Statistical analysis}

Data are presented as mean \pm standard error. Statistical significance was assessed by Wilcoxon signed rank tests with IBM SPSS Statistics 22 software.

\section{Results}

\section{Motor scores}

Individual hand motor score results are provided in Supplementary Table 1 (see online supplementary material at http://www .liebertpub.com). Immediately after the intervention, the motor scores of both hands increased significantly. The PAS hand improved by $0.74 \pm 0.18$ points $(p<0.0001, n=100$ muscles from 5 patients), whereas the PNS hand improved by $0.55 \pm 0.08$ points ( $p<0.0001, n=107$ muscles from 5 patients). There was no significant difference between the improvement in PAS and PNS hands (PAS/PNS: $142 \pm 41 \%, p=0.5, n=5$ patients). During follow-up, however, the PAS hand significantly improved further by $0.37 \pm 0.08$ points ( $p=0.007, n=100$ muscles from 5 patients), whereas there was no significant increase in the motor score in the PNS hand $(0.11 \pm 0.08, p=0.55, n=107$ muscles from 5 patients). The improvement during follow-up was significantly higher in the PAS hand (Fig. 2, PAS/PNS: $228 \pm 28 \%, p=0.042, n=5$ patients). At the third evaluation, the total improvement (third vs. first evaluation) was significantly higher in the PAS than in the PNS hand (Fig. 2, $176 \pm 29 \%, p=0.046, n=5$ patients). Total improvement (third vs. first evaluation) in the PAS hand was $1.02 \pm 0.17$ points $(p<0.0001$, $n=100$ muscles from 5 patients) and in the PNS hand $0.65 \pm 0.14$ points ( $p<0.0001, n=107$ muscles from 5 patients). The motor scores of neck, body, and leg muscles did not significantly improve either at the PAS or at the PNS side (Supplementary Table 2; see online supplementary material at http://www.liebertpub.com).

\section{Sensory scores}

We used the standard scale of American Spinal Injury Association Impairment Scale (AIS) examination to test C2-T10 sensation levels before and immediately after the intervention. The sum of the score was calculated for each patient. Neither PAS (difference of sums after minus before intervention: light touch $-0.8 \pm 1$ points, $p=0.46$ and pin-prick score $3 \pm 1.8$ points, $p=0.14, n=5$ patients) nor PNS (light touch $1.2 \pm 0.6$ points, $p=0.1$ and pin-prick score $-1.4 \pm 2$ points, $p=0.7, n=5$ patients) had an effect on sensory scores.

\section{Spasticity}

Modified Asworth Scale. Spasticity was assessed before, immediately after, and 1 month after the intervention. The difference in points for each joint was calculated similarly to the motor score evaluation. There was no effect of PAS or PNS (Supplementary Table 3; see online supplementary material at http://www .liebertpub.com).

EMG. The number of spasticity-related spikes above the EMG baseline from APB, ADM, and BR muscles did not change significantly by the intervention. The number of spasticity-related spikes after the intervention decreased by $33 \pm 35$ spikes in the PAS hand ( $p=0.24, \mathrm{n}=12$ measurements) and by $23 \pm 16$ spikes in the PNS hand ( $p=0.4, \mathrm{n}=12$ measurements).

\section{F-responses}

We analyzed minimum and maximum $F$ latencies ( $F$ min, F max), $\mathrm{F}$ amplitudes, and $\mathrm{F}$ persistence (pulses that generated F-responses, $\%$ of total amount) from ulnar and median nerves of every patient before and immediately after the intervention. PAS or PNS did not affect these parameters. The average change of median and ulnar nerve measurements by the intervention $(n=10$ measurements from 5 patients) was $\mathrm{F}$ min PAS $-0.14 \pm 0.8 \mathrm{msec}, p=0.44$ and PNS $0.44 \pm 0.78 \mathrm{msec}, p=0.86$, F max PAS $-0.97 \pm 1.5 \mathrm{msec}, p=0.8$ and PNS $-0.39 \pm 0.6 \mathrm{msec}, p=0.33$, F amplitudes PAS $0.18 \pm 0.1 \mathrm{mV}$, $p=0.16$ and PNS $0.07 \pm 0.07 \mathrm{mV}, p=0.1$, F persistence PAS $1 \pm 5 \%$, $p=0.5$, and PNS $-3 \pm 3 \%, p=0.32$.

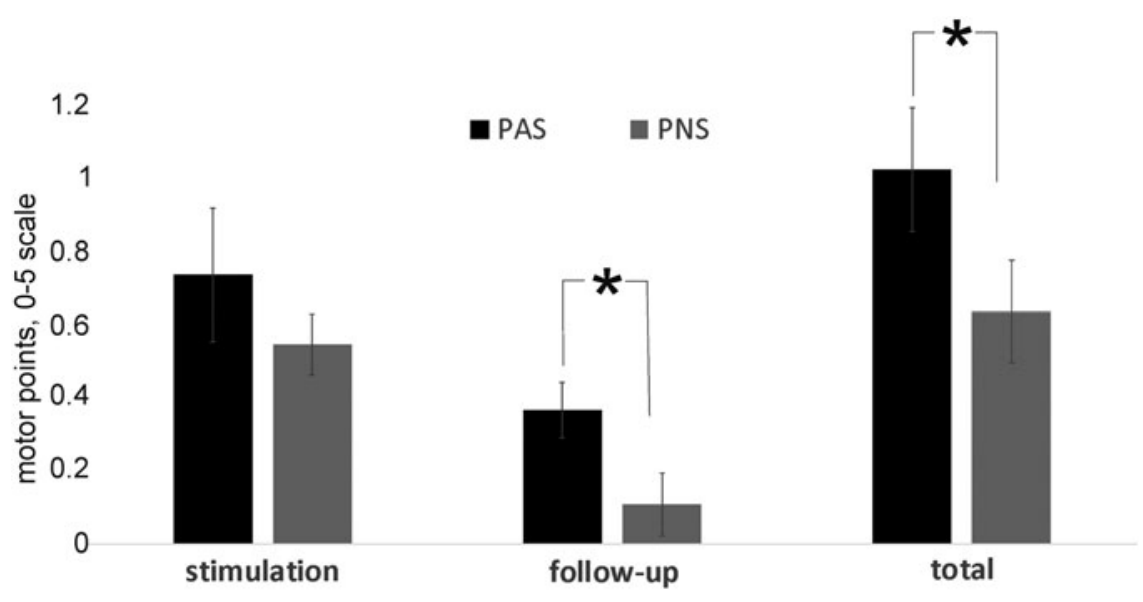

FIG. 2. Improvement in the PAS-treated and PNS-treated hand during stimulation (second vs. first evaluation), follow-up period (third vs. second evaluation), and in total (third vs. first evaluation). PAS, paired associative stimulation; PNS, peripheral nerve stimulation. 


\section{Neuropathic pain}

Patients 2, 3, and 4 reported no neuropathic pain before or after the intervention. Patient 1 reported constant unpleasant tingling in both arms and feet; the tingling was absent after the intervention. Patient 5 reported bilateral constant pain between the elbows and wrists ( $1 \mathrm{~h}$ daily, visual analog scale [VAS] 3) before the intervention. After the intervention, she experienced approximately similar pain ( 1 h daily VAS $4-5$ ). This increase was probably due to interruption of local peripheral stimulation to the wrists (see Methods). After the follow-up period the local peripheral stimulation was resumed, and the intensity of pain returned to VAS 3.

\section{Autonomic functions}

There were no effects on autonomic functions.

\section{Adverse effects}

Patients 1 and 2 reported that it was difficult to sit in the same position during the 2 -h session. The seating was improved and Patients 3-5 reported no such problems.

\section{Discussion}

The effect of long-term PAS on the hand motor output in 5 chronic tetraplegic patients was therapeutic. After 4 weeks of stimulation and 1-month follow-up, each deficient muscle in the PAS-treated hand improved on average by 1 point on a $0-5$ scale. Long-term PAS was more effective than long-term PNS. This is the first sham-controlled study on long-term PAS in SCI patients, and first direct comparison of long-term PAS with long-term unpaired peripheral stimulation in any neurological patient group.

The observed therapeutic effect was not due to alleviation of spasticity. The unchanged F-response parameters indicate that the effect was also not due to changes in peripheral nerves. The most dramatic difference between the PAS- and PNS-treated hands occurred during the follow-up period, when the PAS hand continued to improve, whereas the PNS hand did not. PAS is thought to induce LTP-like plasticity at the targeted synapses. ${ }^{9}$ Evidence derived from in vitro studies and animal experiments indicates that correlated pre-and post-synaptic spiking releases neurotrophins that can contribute to structural changes enhancing the transmission between pre- and post-synaptic neurons. Formation of new synapses, growth of new dendritic spines, and cytoskeletal reorganization can be induced by neurotrophins. These changes are sometimes referred as being part of the "late LTP" and require long-term activation. ${ }^{27,28}$ The plausible mechanism of long-term PAS action is the structural reorganization at the corticomotoneuronal synapses of the cervical spinal cord. The innervation strengthened by PAS possibly enabled the hands to improve beyond the stimulation period as a result from increased voluntary activity and possibly also by trophic support to muscles. ${ }^{29}$ Further research, however, is needed to elucidate to which extent the cortical versus spinal mechanisms are involved in the recovery process.

PNS induced contractions of the muscles innervated by the stimulated nerve. It is plausible that PNS-induced improvement was due to training of the muscles, which was absent after the stimulation was discontinued. This is consistent with our recent observation in healthy subjects, where a PAS protocol identical to the one used here enhanced MEPs, whereas PNS did not. ${ }^{23}$ The therapeutic effect of PAS presumably comprises both muscle training by the PNS component and the strengthening of neural connections. It remains to be determined whether the difference between PAS and PNS-treated hands would be greater if the stimulation lasted longer than 4 weeks.

In our pilot study, ${ }^{20}$ we administered PNS-only to one nerve of one patient and did not observe the effect of the PNS. Here, PNS was given to three nerves and produced a statistically significant effect at the group level. It is noteworthy that although various peripheral stimulation techniques are widely used in rehabilitation after SCI, the settings of PNS we used here are not in routine clinical use. The potential of this protocol in rehabilitation could be of clinical interest by itself in patients having contraindications to TMS.

We cannot rule out the effect of TMS on the contralateral hemisphere. ${ }^{30}$ Nevertheless, because the hands were not stimulated simultaneously but in sequential order, there is no possibility that the PNS hand would have received any external paired stimuli. Similarly to PAS, we also combined PNS with motor imagery to isolate the effect of TMS. Therefore, at the PNS side some motor cortex activation also occurred simultaneously with the electrical stimulation, although it was clearly different from TMS. The motor areas of both hemispheres interact in healthy individuals and in stroke patients. ${ }^{31}$ Accordingly, it is not known whether the improvement of the PAS hand could have contributed to the improvement of the PNS hand. It remains to be determined whether improvement in one hand by PAS would lead to improvement in the other hand if the other hand was untreated. Comparing the hands of the same patient is advantageous as it circumvents the heterogeneity of SCI patients. Both hands of a single patient are also under the influence of identical medication, genetic factors, duration since injury, type of injury, and other patient-specific traits. Importantly, the superiority of the outcome in the PAS versus PNS hand was observed in all patients, regardless of whether the PAS hand was dominant or non-dominant (Table 2), and regardless of whether the PAS hand was weaker or stronger at the initial evaluation (Table 2 and Supplementary Table 1; see online supplementary material at http://www.liebertpub.com).

The treatment did not induce adverse effects. All patients tolerated the intervention well and were motivated to fully adhere to the protocol. The age range of the patients extended up to 68 years, which is important as incidence of SCI in the older population is increasing. ${ }^{32}$

The benefit of the treatment was not only objectively determined by our measurements, but also perceived by the patients. All 5 patients reported that their hand movements improved already before the test results. Functional improvements of daily activities were reported as well. Patients with high baseline motor score before the treatment achieved more functional benefit than the ones with lower scores. For example, Patient 1 became able to open bottles and Patient 3 became able to open doors with the PAStreated hand. These regained skills remained during the follow-up period. The patient with the lowest baseline score did not achieve functional benefits; nevertheless, she was satisfied with the improved ability to move the hands. Four weeks is, however, most probably too short a period to unveil the full potential of the method to induce functional improvements. We propose that the long-term PAS protocol presented here is a useful tool for rehabilitation of SCI. Its properties and therapeutic potential should be investigated in a larger cohort of different types of SCI patients, using longer periods of stimulation, and starting early after SCI.

\section{Acknowledgments}

We are deeply grateful to our patients for participating in the study. We thank David Montero-Danger for excellent technical assistance (MRI) and Andrey Rodionov for cross-checking the data. 
The research was supported in part by the Finnish Cultural Foundation (AT and AS), the Maire Taponen Foundation (AS), the Helsinki University Hospital governmental subsidiary funds for clinical research (AS), the Emil Aaltonen Foundation (AS), and the Faculty of Medicine, University of Helsinki (AS).

\section{Author Disclosure Statement}

PL reports personal fees from Nexstim Ltd. outside the submitted work. Other authors have nothing to report; no competing financial interests exist.

\section{References}

1. Ackery, A., Tator, C., and Krassioukov, A. (2004). A global perspective on spinal cord injury epidemiology. J. Neurotrauma 21, $1355-1370$.

2. Squair, J.W., Bjerkefors, A., Inglis, J.T., Lam, T., and Carpenter, M.G. (2016). Cortical and vestibular stimulation reveal preserved descending motor pathways in individuals with motor-complete spinal cord injury. J. Rehabil. Med. 48, 589-596.

3. Field-Fote, E.C. (2015). Exciting recovery: augmenting practice with stimulation to optimize outcomes after spinal cord injury. Prog. Brain Res. 218, 103-126.

4. Field-Fote, E.C., Yang, J.F., Basso, D.M., and Gorassini, M.A. (2017). Supraspinal control predicts locomotor function and forecasts responsiveness to training after spinal cord injury. J. Neurotrauma 34 , $1813-1825$.

5. Nicoll, R.A. (2017). A brief history of long-term potentiation. Neuron 93, 281-290.

6. McPherson, J.G., Miller, R.R., and Perlmutter, S.I. (2015). Targeted, activity-dependent spinal stimulation produces long-lasting motor recovery in chronic cervical spinal cord injury. Proc. Natl. Acad. Sci. U. S. A. $112,12193-12198$

7. Nishimura, Y., Perlmutter, S.I., Eaton, R.W., and Fetz, E.E. (2013). Spike-timing-dependent plasticity in primate corticospinal connections induced during free behavior. Neuron 80, 1301-1309.

8. Ahmed, Z. (2013). Electrophysiological characterization of spinosciatic and cortico-sciatic associative plasticity: modulation by transspinal direct current and effects on recovery after spinal cord injury in mice. J. Neurosci. 33, 4935-4946.

9. Stefan, K., Kunesch, E., Cohen, L.G., Benecke, R., and Classen, J. (2000). Induction of plasticity in the human motor cortex by paired associative stimulation. Brain 123, Pt. 3, 572-584.

10. Wolters, A., Sandbrink, F., Schlottmann, A., Kunesch, E., Stefan, K., Cohen, L.G., Benecke, R., and Classen, J. (2003). A temporally asymmetric Hebbian rule governing plasticity in the human motor cortex. J. Neurophysiol. 89, 2339-2345.

11. Wolters, A., Schmidt, A., Schramm, A., Zeller, D., Naumann, M., Kunesch, E., Benecke, R., Reiners, K., and Classen, J. (2005). Timingdependent plasticity in human primary somatosensory cortex. J. Physiol. 565, 1039-1052.

12. Carson, R.G., and Kennedy, N.C. (2013). Modulation of human corticospinal excitability by paired associative stimulation. Front. Hum. Neurosci. 7, 823 .

13. Taylor, J.L., and Martin, P.G. (2009). Voluntary motor output is altered by spike-timing-dependent changes in the human corticospinal pathway. J. Neurosci. 29, 11708-11716.

14. Bunday, K.L., and Perez, M.A. (2012). Motor recovery after spinal cord injury enhanced by strengthening corticospinal synaptic transmission. Curr. Biol. 22, 2355-2361.

15. Gordon, T. (2016). Electrical stimulation to enhance axon regeneration after peripheral nerve injuries in animal models and humans. Neurotherapeutics 13, 295-310.
16. Chakravarthy, K., Nava, A., Christo, P.J., and Williams, K. (2016). Review of recent advances in peripheral nerve stimulation (PNS). Curr. Pain Headache Rep. 20, 60.

17. Rossi, S., Hallett, M., Rossini, P.M., Pascual-Leone, A., and Safety of TMS Consensus Group. (2009). Safety, ethical considerations, and application guidelines for the use of transcranial magnetic stimulation in clinical practice and research. Clin. Neurophysiol. 120, 2008-2039.

18. Doucet, B.M., Lam, A., and Griffin, L. (2012). Neuromuscular electrical stimulation for skeletal muscle function. Yale J. Biol. Med. 85, 201-215.

19. Harvey, L.A., Glinsky, J.V., and Bowden, J.L. (2016). The effectiveness of 22 commonly administered physiotherapy interventions for people with spinal cord injury: a systematic review. Spinal Cord 54, 914-923.

20. Shulga, A., Lioumis, P., Zubareva, A., Brandstack, N., Kuusela, L., Kirveskari, E., Savolainen, S., Ylinen, A., and Makela, J.P. (2016). Long-term paired associative stimulation can restore voluntary control over paralyzed muscles in incomplete chronic spinal cord injury patients. Spinal Cord Ser. Cases 2, 16016.

21. Uy, J., Ridding, M.C., Hillier, S., Thompson, P.D., and Miles, T.S. (2003). Does induction of plastic change in motor cortex improve leg function after stroke? Neurology 61, 982-984.

22. Harel, N.Y., and Carmel, J.B. (2016). Paired stimulation to promote lasting augmentation of corticospinal circuits. Neural Plast. 2016, 7043767 [Epub 2016].

23. Shulga, A., Zubareva, A., Lioumis, P., and Makela, J.P. (2016). Paired associative stimulation with high-frequency peripheral component leads to enhancement of corticospinal transmission at wide range of interstimulus intervals. Front. Hum. Neurosci. 10, 470.

24. Hislop, H.J., Avers, D., and Brown, M. (2014). Daniels and Worthingham's Muscle Testing: Techniques of Manual Examination and Performance Testing, 9th ed. Elsevier: p. 528.

25. Shulga, A., Lioumis, P., Kirveskari, E., Savolainen, S., Makela, J.P., and Ylinen, A. (2015). The use of F-response in defining interstimulus intervals appropriate for LTP-like plasticity induction in lower limb spinal paired associative stimulation. J. Neurosci. Methods 242C, 112-117.

26. Di Lazzaro, V., Ziemann, U., and Lemon, R.N. (2008). State of the art: physiology of transcranial motor cortex stimulation. Brain Stimul. 1, 345-362.

27. Poo, M.M. (2001). Neurotrophins as synaptic modulators. Nat. Rev. Neurosci. 2, 24-32.

28. Malenka, R.C., and Bear, M.F. (2004). LTP and LTD: an embarrassment of riches. Neuron 44, 5-21.

29. Midrio, M. (2006). The denervated muscle: facts and hypotheses. A historical review. Eur. J. Appl. Physiol. 98, 1-21.

30. Blankenburg, F., Ruff, C.C., Bestmann, S., Bjoertomt, O., Eshel, N., Josephs, O., Weiskopf, N., and Driver, J. (2008). Interhemispheric effect of parietal TMS on somatosensory response confirmed directly with concurrent TMS-fMRI. J. Neurosci. 28, 13202-13208.

31. Ward, N.S., and Cohen, L.G. (2004). Mechanisms underlying recovery of motor function after stroke. Arch. Neurol. 61, 1844-1848.

32. van den Berg, M.E., Castellote, J.M., Mahillo-Fernandez, I., and de Pedro-Cuesta, J. (2010). Incidence of spinal cord injury worldwide: a systematic review. Neuroepidemiology 34, 184-192; discussion 192.

Address correspondence to: Anastasia Shulga, MD, PhD

BioMag Laboratory

HUS Medical Imaging Center

Helsinki University Central Hospital

PO Box 340

FI-00029 HUS

Finland

E-mail: anastasia.shulga@helsinki.fi 